\title{
Contents
}

Preface

vii

\section{Nonlinear Least Squares}

1 Nonlinear Inverse Problems: Examples and Difficulties 5

1.1 Example 1: Inversion of Knott-Zoeppritz Equations . . . . . . 6

1.2 An Abstract NLS Inverse Problem . . . . . . . . . . . . . . 9 9

1.3 Analysis of NLS Problems . . . . . . . . . . . . . 10

1.3 .1 Wellposedness . . . . . . . . . . . . 10

$1.3 .2 \quad$ Optimizability . . . . . . . . . . . . . . . . . 12

1.3.3 Output Least Squares Identifiability and Quadratically Wellposed Problems . . . . . . . . . . . . . . . 12

1.3.4 Regularization . . . . . . . . . . . . . . . . 14

1.3.5 Derivation . . . . . . . . . . . . . . . 20

1.4 Example 2: 1D Elliptic Parameter Estimation Problem . . . . 21

1.5 Example 3: 2D Elliptic Nonlinear Source Estimation Problem 24

1.6 Example 4: 2D Elliptic Parameter Estimation Problem . . . . 26

2 Computing Derivatives $\quad 29$

2.1 Setting the Scene . . . . . . . . . . . . . . . 30

2.2 The Sensitivity Functions Approach . . . . . . . . . . . . 33

2.3 The Adjoint Approach . . . . . . . . . . . . . . . . . 33

2.4 Implementation of the Adjoint Approach . . . . . . . . . 38

2.5 Example 1: The Adjoint Knott-Zoeppritz Equations . . . . . . 41 
2.6 Examples 3 and 4: Discrete Adjoint Equations . . . . . . . . . 46

2.6.1 Discretization Step 1: Choice of a Discretized Forward Map . . . . . . . . . . . . . . . . . . 47

2.6.2 Discretization Step 2: Choice of a Discretized Objective Function . . . . . . . . . . . . . . . 52

2.6.3 Derivation Step 0: Forward Map and Objective Function 52

2.6.4 Derivation Step 1: State-Space Decomposition . . . . . 53

2.6.5 Derivation Step 2: Lagrangian . . . . . . . . . . . . 54

2.6.6 Derivation Step 3: Adjoint Equation . . . . . . . . . 56

2.6.7 Derivation Step 4: Gradient Equation . . . . . . . . 58

2.7 Examples 3 and 4: Continuous Adjoint Equations . . . . . . . 59

2.8 Example 5: Differential Equations, Discretized Versus

Discrete Gradient . . . . . . . . . . . . . . . . 65

2.8.1 Implementing the Discretized Gradient . . . . . . . 68

2.8.2 Implementing the Discrete Gradient . . . . . . . . . . . 68

2.9 Example 6: Discrete Marching Problems . . . . . . . . . . 73

$\begin{array}{llr}3 & \text { Choosing a Parameterization } & 79\end{array}$

3.1 Calibration ................... . . 80

3.1 .1 On the Parameter Side . . . . . . . . . . 80

3.1 .2 On the Data Side . . . . . . . . . . . . . . . 83

3.1.3 Conclusion . . . . . . . . . . . . . . . 84

3.2 How Many Parameters Can be Retrieved from the Data? . . . 84

3.3 Simulation Versus Optimization Parameters . . . . . . . . . 88

3.4 Parameterization by a Closed Form Formula . . . . . . . . . . 90

3.5 Decomposition on the Singular Basis . . . . . . . . . . . . . 91

3.6 Multiscale Parameterization . . . . . . . . . . . . . . 93

3.6.1 Simulation Parameters for a Distributed Parameter . . 93

3.6.2 Optimization Parameters at Scale $k$. . . . . . . . . 94

3.6.3 Scale-By-Scale Optimization . . . . . . . . . . . . 95

3.6.4 Examples of Multiscale Bases . . . . . . . . . . . . 105

3.6.5 Summary for Multiscale Parameterization . . . . . . . 108

3.7 Adaptive Parameterization: Refinement Indicators . . . . . . . 108

3.7.1 Definition of Refinement Indicators . . . . . . . . . . . 109

3.7.2 Multiscale Refinement Indicators . . . . . . . . . . . 116

3.7.3 Application to Image Segmentation . . . . . . . . . . . 121

3.7.4 Coarsening Indicators . . . . . . . . . . . . . . . . . 122

3.7.5 A Refinement/Coarsening Indicators Algorithm . . . . 124 
3.8 Implementation of the Inversion . . . . . . . . . . . . . 126

3.8.1 Constraints and Optimization Parameters . . . . . 126

3.8.2 Gradient with Respect to Optimization

Parameters . . . . . . . . . . . . . . . 129

3.9 Maximum Projected Curvature: A Descent Step for Nonlinear

Least Squares . . . . . . . . . . . . . . . . 135

3.9.1 Descent Algorithms . . . . . . . . . . . . . . 135

3.9.2 Maximum Projected Curvature (MPC) Step . . . . . . 137

3.9.3 Convergence Properties for the Theoretical

MPC Step . . . . . . . . . . . . . . . . . . . . . 143

3.9.4 Implementation of the MPC Step . . . . . . . . . . . 144

3.9.5 Performance of the MPC Step . . . . . . . . . . . 148

\section{Output Least Squares Identifiability and Quadratically} Wellposed NLS Problems

4.1 The Linear Case . . . . . . . . . . . . . . . . . . . . . . . . . 163

4.2 Finite Curvature/Limited Deflection Problems . . . . . . . . . 165

4.3 Identifiability and Stability of the Linearized Problems . . . . 174

4.4 A Sufficient Condition for OLS-Identifiability . . . . . . . . . 176

4.5 The Case of Finite Dimensional Parameters . . . . . . . . . . 179

4.6 Four Questions to Q-Wellposedness . . . . . . . . . . . . . . . 182

4.6.1 Case of Finite Dimensional Parameters . . . . . . . . . 183

4.6.2 Case of Infinite Dimensional Parameters . . . . . . . . 184

4.7 Answering the Four Questions . . . . . . . . . . . . . . . . . 184

4.8 Application to Example 2: 1D Parameter Estimation with $H^{1}$

Observation . . . . . . . . . . . . . . . 191

4.8.1 Linear Stability . . . . . . . . . . . . . . . . . 193

4.8.2 Deflection Estimate . . . . . . . . . . . . . . . 198

4.8.3 Curvature Estimate . . . . . . . . . . . . . . . 199

4.8.4 Conclusion: OLS-Identifiability . . . . . . . . 200

4.9 Application to Example 4: 2D Parameter Estimation, with $H^{1}$ Observation . . . . . . . . . . . . . . . 200

5 Regularization of Nonlinear Least Squares Problems 209

5.1 Levenberg-Marquardt-Tychonov (LMT)

Regularization ................... 209

5.1 Linear Problems . . . . . . . . . . . . . . 211

5.1.2 Finite Curvature/Limited Deflection

(FC/LD) Problems . . . . . . . . . . . . . . . . 219

5.1.3 General Nonlinear Problems . . . . . . . . . . . . 231 
5.2 Application to the Nonlinear 2D Source Problem . . . . . . 237

5.3 State-Space Regularization . . . . . . . . . . . . . . . . . . 246

5.3.1 Dense Observation: Geometric Approach . . . . . . . . 248

5.3.2 Incomplete Observation: Soft Analysis . . . . . . . . . 256

5.4 Adapted Regularization for Example 4: 2D Parameter

Estimation with $H^{1}$ Observation . . . . . . . . . . . . 259

5.4.1 Which Part of $a$ is Constrained by the Data?. . . . . . 260

5.4.2 How to Control the Unconstrained Part? . . . . . . . . . 262

5.4.3 The Adapted-Regularized Problem . . . . . . . . . . . 264

5.4.4 Infinite Dimensional Linear Stability and Deflection Estimates . . . . . . . . . . . 265

5.4 .5 Finite Curvature Estimate . . . . . . . . . . . 267

5.4.6 OLS-Identifiability for the Adapted Regularized Problem . . . . . . . . . . . . . . . . . 268

\section{A Generalization of Convex Sets}

6 Quasi-Convex Sets

6.1 Equipping the Set $D$ with Paths . . . . . . . . . . . . . 277

6.2 Definition and Main Properties of q.c. Sets . . . . . . . . . . . 281

7 Strictly Quasi-Convex Sets

7.1 Definition and Main Properties of s.q.c. Sets . . . . . . . . . . 300

7.2 Characterization by the Global Radius of Curvature . . . . . . 304

7.3 Formula for the Global Radius of Curvature . . . . . . . . . . 316

8 Deflection Conditions for the Strict Quasi-convexity of Sets

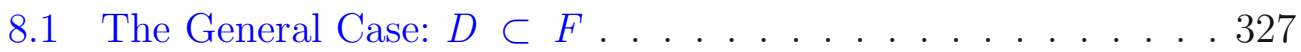

8.2 The Case of an Attainable Set $D=\varphi(C) \ldots 337$

Bibliography

Index 